\title{
POSITIVELY EXPANSIVE MAPS AND GROWTH OF FUNDAMENTAL GROUPS
}

\author{
K. HIRAIDE
}

(Communicated by Kenneth R. Meger)

\begin{abstract}
In this note we will show that a positively expansive map of an arbitrary closed topological manifold is topologically conjugate to an expanding infra-nil-endomorphism.
\end{abstract}

Let $\Gamma$ be a group generated by $\left\{\gamma_{1}, \ldots, \gamma_{k}\right\}$. Then each $\gamma \in \Gamma$ is represented as a word $\gamma_{i_{1}}^{p_{1}} \gamma_{i_{2}}^{p_{2}} \cdots \gamma_{i_{l}}^{p_{l}}$ and the number $\left|p_{1}\right|+\left|p_{2}\right|+\cdots+\left|p_{l}\right|$ is called the length of the word. The norm $\|\gamma\|$ is defined as the minimal length of the word representing $\gamma$. As properties of the norm we know that $\|\gamma\|=\left\|\gamma^{-1}\right\|$ and $\left\|\gamma \gamma^{\prime}\right\| \leq\|\gamma\|+\left\|\gamma^{\prime}\right\|$. If $\delta_{1}, \ldots, \delta_{k}$ is another system of generators in $\Gamma$, then the corresponding norm \|\|$^{\prime}$ is not necessarily equal to \|\| . But there exists $c>0$ such that for each $\gamma \in \Gamma$

$$
c\|\gamma\|^{\prime} \geq\|\gamma\| \geq c^{-1}\|\gamma\|^{\prime} .
$$

Let $\Gamma$ be a finitely generated group and fix its generators. We denote by $B(t)$, $t>0$, the ball of radius $t$ centered at the identity element $e$, and by $\# B(t)$ the number of elements in $B(t)$.

We say that $\Gamma$ has polynomial growth if there are two positive numbers $d$ and $c$ such that for all balls $B(t), t \geq 1, \# B(t) \leq c t^{d}$. If $\Gamma$ has a nilpotent subgroup of finite index then $\Gamma$ has polynomial growth (Wolf $[\mathbf{1 0}]$ ).

The following is one interesting result for our investigation.

THEOREM A (M. GROMOV [5]). If a finitely generated group $\Gamma$ has polynomial growth, then $\Gamma$ contains a nilpotent subgroup of finite index.

Let $L$ be a simply connected nilpotent Lie group with a left invariant Riemannian metric and denote by $\operatorname{Aff}(L)$ the group of transformations of $L$ generated by the left translations and by all automorphisms from $L$ onto itself. Let $\Gamma \subset \operatorname{Aff}(L)$ be a group which acts freely and discretely on $L$. If the quotient $X=L / \Gamma$ is compact, then it is called an infra-nil-manifold. Each expanding automorphism $L \rightarrow L$ which respects $\Gamma$ induces an expanding map $X \rightarrow X$. Such maps are called an expanding infra-nil-endomorphism.

From the following results together with Theorem A, M. Gromov [5] proved that an expanding differentiable map of an arbitrary closed $C^{\infty}$ manifold is topologically conjugate to an infra-nil-endomorphism.

Received by the editors November 2, 1987.

1980 Mathematics Subject Classification (1985 Revision). Primary 54H20, 58F15; Secondary 57 N65.

Key words and phrases. Positively expansive map, expanding infra-nil-endomorphism, growth of fundamental group. 
THEOREM B (M. SHUB [9]). An expanding differentiable map of a closed $C^{\infty}$ manifold $M$ is topologically conjugate to an expanding infra-nil-endomorphism if and only if the fundamental group $\pi_{1}(M)$ contains a nilpotent subgroup of finite index.

THEOREM C (J. FRANKS [4]). If a closed $C^{\infty}$ manifold $M$ admits an expanding differentiable map, then the fundamental group $\pi_{1}(M)$ has polynomial growth.

Our aim is to prove that Gromov's result is true for positively expansive maps being more general than expanding differentiable maps.

A continuous map $f: X \rightarrow X$ of a metric space $(X, d)$ is called positively expansive if there is a constant $c>0$ such that if $x \neq y$ then $d\left(f^{n}(x), f^{n}(y)\right) \geq c$ for some $n \geq 0$. If, in particular, $X$ is a closed topological manifold and $f$ is positively expansive, then $f$ is a covering map with covering degree $\geq 2$.

Our assertion is the following

THEOREM. A positively expansive map of an arbitrary closed topological manifold is topologically conjugate to an expanding infra-nil-endomorphism.

Hereafter, let $M$ be a closed topological manifold and $\pi: \bar{M} \rightarrow M$ be the universal cover. For the proof of our theorem we need the following

LEMMA 1 (E. COVEN AND W. REDDY [2]). If $f: M \rightarrow M$ is a positively expansive map, then there exists a metric $\bar{d}$ for $\bar{M}$ such that

(i) $\bar{d}$ is complete,

(ii) all covering transformations are isometries,

(iii) if $\bar{f}: \bar{M} \rightarrow \bar{M}$ is a lift of $f$ by $\pi$, then there is $\lambda>1$ such that $\bar{d}(\bar{f}(x), \bar{f}(y)) \geq$ $\lambda \bar{d}(x, y)$ for $x, y \in \bar{M}$.

By making use of Shub [8] and Lemma 1, (I) and (II) below are easily obtained (see P. Duvall and L. Husch [3] for the proof of (I)(a) and refer R. Kirby and L. Siebenmann [6] for the proof of (II)):

(I) $[8$, pp. 171-181] If $f: M \rightarrow M$ is a positively expansive map, then

(a) $\bar{M}$ is homeomorphic to $\mathrm{R}^{n}(n=\operatorname{dim}(\bar{M}))$,

(b) $f$ has a fixed point, $M)$,

(c) the nonwandering set of $f, \Omega(f)$, coincides with the whole space (i.e. $\Omega(f)=$

(d) $\pi_{1}(M)$ is torsion free,

(e) $M$ is of type $K(\pi, 1)$.

(II) [8, pp. 181-184] Let $g: N \rightarrow N$ be a continuous map of a compact topological manifold $N$ and have a fixed point $n_{0}$, and let $m_{0}$ be a fixed point of a positively expansive map $f: M \rightarrow M$. If there is a group homomorphism $A: \pi_{1}\left(N, n_{0}\right) \rightarrow$ $\pi_{1}\left(M, m_{0}\right)$ such that the following diagram commutes:

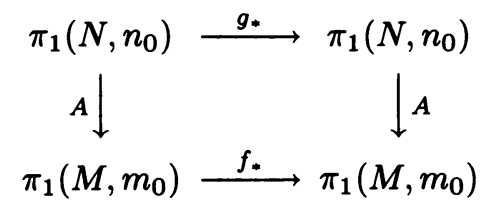

then one finds a unique continuous map $h: N \rightarrow M$ such that $h\left(n_{0}\right)=m_{0}, h_{*}=A$ and $h \circ g=f \circ h$. 
PROPOSITION 2. If $M$ admits a positively expansive map, then the fundamental group $\pi_{1}(M)$ has polynomial growth.

If we establish Proposition 2, then our theorem is proved as follows. Let $f: M \rightarrow$ $M$ be a positively expansive map. Then by (I)(b), $f$ has a fixed point $m_{0}$. By Theorem A, $\pi_{1}(M)$ contains a nilpotent subgroup of finite index. Since $f: M \rightarrow M$ is a covering map and $\pi_{1}(M)$ is torsion free (by $\left.(\mathrm{I})(\mathrm{d})\right)$, by Franks [4, Existence Theorem 3.5], there exist an infra-nil-manifold $L / \Gamma$, an infra-nil-endomorphism $g: L / \Gamma \rightarrow L / \Gamma$ and an isomorphism $A: \pi_{1}(L / \Gamma, e) \rightarrow \pi_{1}\left(M, m_{0}\right)$ such that the following diagram commutes:

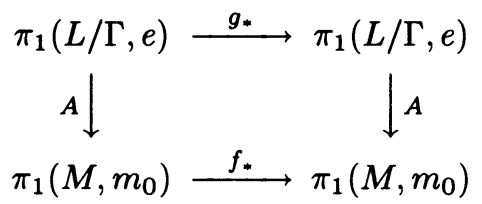

Then by (II) we can find a continuous map $h: L / \Gamma \rightarrow M$ with $h(e)=m_{0}$ such that $h_{*}=A$ and $h \circ g=f \circ h$. Combining this with Lemma 1, by the technique of J. Franks [4, p. 91] we see that $g$ is expanding. Hence there is a continuous map $h^{\prime}: M \rightarrow L / \Gamma$ with $h^{\prime}\left(m_{0}\right)=e$ such that $h_{*}^{\prime}=A^{-1}$ and $h^{\prime} \circ f=g \circ h^{\prime}$ [8, Theorem 4]. Since $h \circ g=f \circ h$, it follows that $h \circ h^{\prime} \circ f=f \circ h \circ h^{\prime}$ and $g \circ h^{\prime} \circ h=h^{\prime} \circ h \circ g$. By uniqueness we obtain that $h \circ h^{\prime}$ and $h^{\prime} \circ h$ are identity maps. The proof of the theorem is completed.

It only remains to prove Proposition 2. To do this, let $d$ be a metric for $M$. Then we know (cf. [3]) that there are a metric $\rho$ for $\bar{M}$ and $\eta_{0}>0$ such that

(1) if $\rho(x, y) \leq \eta_{0}$, then $d(\pi(x), \pi(y))=\rho(x, y)$,

(2) all covering transformations are isometries under $\rho$.

Fix $0<\eta<\eta_{0} / 2$ and let $x, y \in \bar{M}$. By a $\eta$-chain from $x$ to $y$ we mean a finite sequence $\left(x_{0}, \ldots, x_{n}\right)$ of points in $\bar{M}$ such that $x=x_{0}, y=x_{n}$ and $\rho\left(x_{i}, x_{i+1}\right) \leq \eta$ for $0 \leq i \leq n-1$. Define

$$
\tilde{d}(x, y)=\inf \left\{\sum_{i=0}^{m-1} \rho\left(x_{i}, x_{i+1}\right)\right\}
$$

where the infimum is taken over all $\eta$-chains from $x$ to $y$. Then by the triangle inequality we have

$$
\tilde{d}(x, y) \geq \rho(x, y) \text { for all } x, y \in \bar{M},
$$

from which it follows that $\tilde{d}$ is a metric for $\bar{M}$. If $\rho(x, y) \leq \eta$, then by the definition of $\tilde{d}, \tilde{d}(x, y) \leq \rho(x, y)$ and hence $\tilde{d}(x, y)=\rho(x, y)$ (by (3)). Therefore

$$
\rho(x, y) \leq \eta \Rightarrow \tilde{d}(x, y)=\rho(x, y) .
$$

By (2) it is easily checked that

(5) all covering transformations are isometries under $\tilde{d}$.

We can consider that $\pi_{1}(M)$ is the group of all covering transformations. Let $K$ be a compact covering domain for $\pi$ and put

$$
F=\left\{\gamma \in \pi_{1}(M): \tilde{d}(\gamma(K), K) \leq \eta\right\}
$$


where $\tilde{d}(\gamma(K), K)=\min \{\tilde{d}(x, y): x \in \gamma(K), y \in K\}$. Then $F$ is finite. For, if not, there is an infinite subset $\left\{\gamma_{i}\right\}_{i \in \mathbf{N}} \subset \pi_{1}(M)$ such that $\tilde{d}\left(\gamma_{i}(K), K\right) \leq \eta$. Take $y_{i} \in \gamma_{i}(K)$ such that $\tilde{d}\left(y_{i}, K\right) \leq \eta$. Since $\gamma_{i}^{-1}\left(y_{i}\right) \in K$ and $K$ is compact, we may assume that $\gamma_{i}^{-1}\left(y_{i}\right)$ converges to some $y_{\infty} \in K$ as $i \rightarrow \infty$. If $\tilde{d}\left(\gamma_{i}\left(y_{\infty}\right), \gamma_{j}\left(y_{\infty}\right)\right) \leq$ $\eta_{0}$, then

$$
\begin{aligned}
\tilde{d}\left(\gamma_{i}\left(y_{\infty}\right), \gamma_{j}\left(y_{\infty}\right)\right) & \geq \rho\left(\gamma_{i}\left(y_{\infty}\right), \gamma_{j}\left(y_{\infty}\right)\right) \quad(\text { by }(3)) \\
& =d\left(\pi \circ \gamma_{i}\left(y_{\infty}\right), \pi \circ \gamma_{j}\left(y_{\infty}\right)\right) \quad(\text { by }(1)) \\
& =d\left(\pi\left(y_{\infty}\right), \pi\left(y_{\infty}\right)\right)=0,
\end{aligned}
$$

and so $\rho\left(\gamma_{i}\left(y_{\infty}\right), \gamma_{j}\left(y_{\infty}\right)\right)=0$, from which $\gamma_{i}=\gamma_{j}$. Hence $\tilde{d}\left(\gamma_{i}\left(y_{\infty}\right), \gamma_{j}\left(y_{\infty}\right)\right)>\eta_{0}$ for all $i \neq j$. Since

$$
\begin{aligned}
\tilde{d}\left(\gamma_{i}\left(y_{\infty}\right), y_{i}\right) & =\tilde{d}\left(y_{\infty}, \gamma_{i}^{-1}\left(y_{i}\right)\right) \quad(\text { by }(5)) \\
& \rightarrow 0 \quad \text { as } i \rightarrow \infty
\end{aligned}
$$

we have $\tilde{d}\left(y_{i}, y_{j}\right)>\eta_{0}$ for sufficiently large $i \neq j$. On the other hand, since $\tilde{d}\left(y_{i}, K\right) \leq \eta$, there is $z_{i} \in K$ such that $\tilde{d}\left(z_{i}, y_{i}\right) \leq \eta$. Since $K$ is compact, we may assume that $z_{i}$ converges to some point in $K$. Then for $\varepsilon>0$ there is $i_{0}>0$ such that $\tilde{d}\left(y_{i}, y_{j}\right) \leq 2 \eta+\varepsilon$ for $i, j \geq i_{0}$, which contradicts $2 \eta<\eta_{0}$. Therefore $F$ is finite.

Since $\pi_{1}(M)$ is finitely generated, we may assume $F$ is a system of generators in $\pi_{1}(M)$ (choose suitably a finite subset of $\pi_{1}(M)$ and take a union of it and $F$ if necessary). We write

$$
\nu=\inf \left\{\tilde{d}(\gamma(K), K): \gamma \in \pi_{1}(M), \gamma \notin F\right\} .
$$

By the choice of $F$ it follows that $\nu \geq \eta$. For fixed $y_{0} \in K$ write

$$
\mu=\max \left\{\tilde{d}\left(\gamma\left(y_{0}\right), y_{0}\right): \gamma \in F\right\} .
$$
[7]).

Claim 1. If $\tilde{d}\left(y_{0}, \gamma(K)\right) \leq \nu t$ for $t \geq 0$, then $\|\gamma\| \leq 2 t+2$ (compare with Milnor

Let $t \geq 0$ and choose $y \in \gamma(K)$ such that $\tilde{d}\left(y_{0}, y\right) \leq \nu t$. By the definition of $\tilde{d}$, for any $\varepsilon>0$ there is a $\eta$-chain $\left(x_{0}^{\prime}, \ldots, x_{n}^{\prime}\right)$ from $y_{0}$ to $y$ such that

$$
\sum_{i=0}^{n-1} \rho\left(x_{i}^{\prime}, x_{i+1}^{\prime}\right)-\varepsilon \leq \tilde{d}\left(y_{0}, y\right) \text {. }
$$

From (4) together with $\nu \geq \eta$ it follows that $\left(x_{0}^{\prime}, \ldots, x_{n}^{\prime}\right)$ is a $\nu$-chain from $y_{0}$ to $y$ with respect to $\tilde{d}$ and

$$
\sum_{i=0}^{n-1} \tilde{d}\left(x_{i}^{\prime}, x_{i+1}^{\prime}\right)-\varepsilon \leq \tilde{d}\left(y_{0}, y\right)
$$

In the case when $\tilde{d}\left(x_{i}^{\prime}, x_{i+1}^{\prime}\right)+\cdots+\tilde{d}\left(x_{i+j-1}^{\prime}, x_{i+j}^{\prime}\right) \leq \nu$, let us take $\left(x_{i+1}^{\prime}, \ldots, x_{i+j-1}^{\prime}\right)$ away from $\left(x_{0}^{\prime}, \ldots, x_{n}^{\prime}\right)$. Then we have a $\nu$-chain $\left(x_{0}, \ldots, x_{m}\right)$ from $y_{0}$ to $y$ with respect to $\tilde{d}$ such that

$$
\begin{gathered}
\sum_{i=0}^{m-1} \tilde{d}\left(x_{i}, x_{i+1}\right)-\varepsilon \leq \tilde{d}\left(y_{0}, y\right), \\
\nu<\tilde{d}\left(x_{i}, x_{i+1}\right)+\tilde{d}\left(x_{i+1}, x_{i+2}\right) \quad(0 \leq i \leq m-2) .
\end{gathered}
$$


These two inequalities yield

$$
\nu(m / 2-1) \leq \tilde{d}\left(y_{0}, y\right)+\varepsilon
$$

and hence

$$
m \leq(2 / \nu)\left\{\tilde{d}\left(y_{0}, y\right)+\varepsilon\right\}+2 \leq 2 t-2 \varepsilon / \nu+2 .
$$

Since $K$ is a covering domain, we can find $h_{i} \in \pi_{1}(M)$ such that $x_{i} \in h_{i}(K)$ for $0 \leq i \leq m$. Put $h_{0}=$ id and $h_{m}=\gamma$. Since each $h_{i}$ is an isometry (by (5)), we have

$$
\tilde{d}\left(h_{i}^{-1} \circ h_{i+1}(K), K\right)=\tilde{d}\left(h_{i+1}(K), h_{i}(K)\right) \leq \tilde{d}\left(x_{i+1}, x_{i}\right) \leq \nu .
$$

Hence there is $f_{i} \in F$ such that $h_{i}^{-1} \circ h_{i+1}=f_{i}(0 \leq i \leq m-1)$, and so

$$
\begin{aligned}
\gamma & =\left(h_{0}^{-1} \circ h_{1}\right) \circ\left(h_{1}^{-1} \circ h_{2}\right) \circ \cdots \circ\left(h_{m-1}^{-1} \circ h_{m}\right) \\
& =f_{0} \circ \cdots \circ f_{m-1},
\end{aligned}
$$

from which $\|\gamma\| \leq 2 t-2 \varepsilon / \nu+2$. Since $\varepsilon$ is arbitrary, Claim 1 holds.

Claim 2. If $\|\gamma\| \leq t$, then $\tilde{d}\left(y_{0}, \gamma\left(y_{0}\right)\right) \leq \mu t$.

Write $\gamma=f_{1} \circ f_{2} \circ \cdots \circ f_{s}(s \leq t)$ where $f_{i} \in F$ for $1 \leq i \leq s$. Then we have

$$
\begin{aligned}
\tilde{d}\left(y_{0}, \gamma\left(y_{0}\right)\right) \leq & \tilde{d}\left(y_{0}, f_{1}\left(y_{0}\right)\right)+\tilde{d}\left(f_{1}\left(y_{0}\right), f_{1} \circ f_{2}\left(y_{0}\right)\right) \\
& +\cdots+\tilde{d}\left(f_{1} \circ \cdots \circ f_{s-1}\left(y_{0}\right), f_{1} \circ \cdots \circ f_{s}\left(y_{0}\right)\right) \\
= & \tilde{d}\left(y_{0}, f_{1}\left(y_{0}\right)\right)+\tilde{d}\left(y_{0}, f_{2}\left(y_{0}\right)\right)+\cdots+\tilde{d}\left(y_{0}, f_{s}\left(y_{0}\right)\right) \\
\leq & \mu s \leq \mu t .
\end{aligned}
$$

Let $\Gamma=\left\{\gamma\left(y_{0}\right): \gamma \in \pi_{1}(M)\right\}$ and put $\widetilde{B}(t)=\Gamma \cap \widetilde{B}_{t}\left(y_{0}\right)$ where $\widetilde{B}_{t}\left(y_{0}\right)=\{y \in$ $\left.\bar{M}: \tilde{d}\left(y_{0}, y\right) \leq t\right\}$. By Claims 1 and 2 we have

$$
\# \widetilde{B}(2 \mu t+2 \mu) \geq \# B(2 t+2) \geq \# \widetilde{B}(\nu t) \quad(t \geq 0)
$$

and hence letting $c=\max \left\{\mu, \nu / 2, \mu^{-1},(\nu / 2)^{-1}\right\}$,

$$
\# \widetilde{B}(c t) \geq \# B(t) \geq \# \widetilde{B}\left(c^{-1}(t-2)\right) \quad(t \geq 2) .
$$

Take a compact subset $D$ and $s>0$, and define

$$
M_{s}(D)=\max \{\# E: E \in \mathscr{S}(D, s)\}
$$

where

$$
\mathscr{S}(D, s)=\{E \subset D: x, y \in E(x \neq y) \Rightarrow \tilde{d}(x, y) \geq s\} .
$$

Then clearly $M_{s_{1}}(D) \geq M_{s_{2}}(D)$ if $s_{1} \leq s_{2}$. Let $t \geq 0$ and $x \in \bar{M}$. Then it is checked that $\widetilde{B}_{t}(x)$ is compact. Indeed, let $K$ be as above and let $N_{\eta}(K)=\{y \in$ $\bar{M}: \tilde{d}(y, K) \leq \eta\}$. Then by the choice of $F, N_{\eta}(K) \subset \bigcup_{\gamma \in F} \gamma(K)$. Since $F$ is finite, $N_{\eta}(K)$ is compact. Note that $N_{\eta}(K)$ also is a covering domain for $\pi$. Then we have $N_{2 \eta}(K)=N_{\eta}\left(N_{\eta}(K)\right)$ is compact, and so is $N_{n \eta}(K)$ for all $n \in \mathbf{N}$. Since $\widetilde{B}_{t}(x) \subset N_{n \eta}(K)$ for some $n, \widetilde{B}_{t}(x)$ is compact.

Claim 3. $M_{\eta}\left(\widetilde{B}_{t}\left(y_{0}\right)\right) \geq \# \widetilde{B}(t)$ for $t \geq 0$.

Take $x, y \in \widetilde{B}(t)$ and let $\tilde{d}(x, y) \leq \eta$. Then $\rho(x, y) \leq \eta$ by (3), and so by (1)

$$
\rho(x, y)=d(\pi(x), \pi(y))=d\left(\pi\left(y_{0}\right), \pi\left(y_{0}\right)\right)=0 .
$$

Hence $x=y$. This implies $\widetilde{B}(t) \in \mathscr{S}\left(\widetilde{B}_{t}\left(y_{0}\right), \eta\right)$. Therefore Claim 3 holds. 
Let $K$ be as above and let $c_{1}$ be the diameter of $K$. Then we have

Claim 4. \# $\widetilde{B}\left(t+c_{1}\right) \geq M_{3 c_{1}}\left(\widetilde{B}_{t}\left(y_{0}\right)\right)$ for $t \geq 0$.

Choose $E \in \mathscr{S}\left(\widetilde{B}_{t}\left(y_{0}\right), 3 c_{1}\right)$ such that $\# E=M_{3 c_{1}}\left(\widetilde{B}_{t}\left(y_{0}\right)\right)$. For $x, y \in E$ with $x \neq y$ we have $\widetilde{B}_{c_{1}}(x) \cap \widetilde{B}_{c_{1}}(y)=\varnothing$. Since $K$ is a covering domain, there are $\gamma_{x}, \gamma_{y} \in \pi_{1}(M)$ such that $x \in \gamma_{x}(K)$ and $y \in \gamma_{y}(K)$. By (5) and the choice of $c_{1}, \gamma_{x}(K) \subset \widetilde{B}_{c_{1}}(x)$ and $\gamma_{y}(K) \subset \widetilde{B}_{c_{1}}(y)$. Hence $\gamma_{x}(K) \cap \gamma_{y}(K)=\varnothing$ and so $\gamma_{x}\left(y_{0}\right) \neq \gamma_{y}\left(y_{0}\right)$. Since $\widetilde{B}_{c_{1}}(x)$ and $\widetilde{B}_{c_{1}}(y)$ are contained in $B_{t+c_{1}}\left(y_{0}\right)$, we have $\gamma_{x}\left(y_{0}\right), \gamma_{y}\left(y_{0}\right) \in \widetilde{B}\left(t+c_{1}\right)$. The conclusion is obtained from the existence of the injection $E \rightarrow \widetilde{B}\left(t+c_{1}\right)$.

Let $\delta=\min \left\{\eta, 3 c_{1}, \eta^{-1},\left(3 c_{1}\right)^{-1}\right\}$. Then (6) and Claims 3 and 4 tell us that

$$
M_{\delta}\left(\widetilde{B}_{c t}\left(y_{0}\right)\right) \geq \# B(t) \geq M_{\delta^{-1}}\left(\widetilde{B}_{c^{-1}(t-2)-c_{1}}\left(y_{0}\right)\right) \quad\left(t \geq c c_{1}+2\right) .
$$

Now we are ready to prove Proposition 2. Let $f: M \rightarrow M$ be a positively expansive map and $\bar{d}$ be as in Lemma 1. For $x, y \in M$ define

$$
d(x, y)=\min \left\{\bar{d}(\bar{x}, \bar{y}): \bar{x} \in \pi^{-1}(x), \bar{y} \in \pi^{-1}(y)\right\} .
$$

Then by Lemma 1 (ii) it is easily checked that $d$ is a metric for $M$ and there is $\eta_{0}>0$ such that $\bar{d}(x, y) \leq \eta_{0}$ implies $d(\pi(x), \pi(y))=\bar{d}(x, y)$. Hence $\bar{d}$ satisfies properties (1) and (2). Fix $0<\eta<\eta_{0} / 2$ and define the metric $\tilde{d}$ for $\bar{M}$ as above. Let $\bar{f}: \bar{M} \rightarrow \bar{M}$ be the lift of $f$ by $\pi$ so that $\bar{f}\left(y_{0}\right)=y_{0}$ for some $y_{0} \in \bar{M}$ (see (I)(b)). Then we can find $\lambda>1$ such that $\tilde{d}(\bar{f}(x), \bar{f}(y)) \geq \lambda \tilde{d}(x, y)$ for $x, y \in \bar{M}$. Indeed, for $\varepsilon>0$ there is a $\eta$-chain $\left(x_{0}, \ldots, x_{n}\right)$ from $\bar{f}(x)$ to $\bar{f}(y)$ such that

$$
\sum_{i=0}^{n-1} \bar{d}\left(x_{i}, x_{i+1}\right) \leq \tilde{d}(\bar{f}(x), \bar{f}(y))+\varepsilon .
$$

By Lemma 1(iii) there is $\lambda>1$ such that $\bar{d}(\bar{f}(u), \bar{f}(v)) \geq \lambda \bar{d}(u, v)$ for $u, v \in \bar{M}$. Hence $\bar{d}\left(\bar{f}^{-1}\left(x_{i}\right), \bar{f}^{-1}\left(x_{i+1}\right)\right) \leq \lambda^{-1} \bar{d}\left(x_{i}, x_{i+1}\right)<\eta$, and so $\left(\bar{f}^{-1}\left(x_{0}\right), \ldots, \bar{f}^{-1}\left(x_{n}\right)\right)$ is a $\eta$-chain from $x$ to $y$. By this fact we can calculate that

$$
\begin{aligned}
\tilde{d}(x, y) & \leq \sum_{i=0}^{n-1} \bar{d}\left(\bar{f}^{-1}\left(x_{i}\right), \bar{f}^{-1}\left(x_{i+1}\right)\right) \\
& \leq \lambda^{-1} \sum_{i=0}^{n-1} \bar{d}\left(x_{i}, x_{i+1}\right) \\
& \leq \lambda^{-1}(\tilde{d}(\bar{f}(x), \bar{f}(y))+\varepsilon)
\end{aligned}
$$

Since $\varepsilon$ is arbitrary, we conclude that $\tilde{d}(\bar{f}(x), \bar{f}(y)) \geq \lambda \tilde{d}(x, y)$.

Since $\bar{f}\left(y_{0}\right)=y_{0}$, by the above result $\bar{f}\left(\widetilde{B}_{t}\left(y_{0}\right)\right) \supset \widetilde{B}_{\lambda t}\left(y_{0}\right)$ for $t \geq 0$.

Let $K$ be as above and let $N_{\delta}(K)=\{y \in \bar{M}: \tilde{d}(y, K) \leq \delta\}$. As we saw before $N_{\delta}(K)$ is compact and hence

$$
A=\max \left\{\tilde{d}(\bar{f}(x), \bar{f}(y)): x, y \in N_{\delta}(K)\right\}
$$


exists. If $\tilde{d}(x, y) \leq \delta$, then there is $\gamma \in \pi_{1}(M)$ such that $\gamma(x), \gamma(y) \in N_{\delta}(K)$, and so

$$
\begin{aligned}
A & \geq \tilde{d}(\bar{f} \circ \gamma(x), \bar{f} \circ \gamma(y)) \\
& =\tilde{d}\left(\gamma^{\prime} \circ \bar{f}(x), \gamma^{\prime} \circ \bar{f}(y)\right) \quad\left(\text { for some } \gamma^{\prime} \in \pi_{1}(M)\right) \\
& =\tilde{d}(\bar{f}(x), \bar{f}(y)) \quad(\text { by }(5)) .
\end{aligned}
$$

Therefore $\tilde{d}(\bar{f}(x), \bar{f}(y)) \geq A$ implies $\tilde{d}(x, y) \geq \delta$. From this fact we see that $M_{A}(\bar{f}(D)) \leq M_{\delta}(D)$ for any compact subset $D$ of $\bar{M}$.

Let $x \in \bar{M}$. Then there is $\gamma \in \pi_{1}(M)$ such that $\gamma \widetilde{B}_{A}(x) \subset N_{A}(K)$. Since $M_{\delta / 3}\left(\gamma \widetilde{B}_{A}(x)\right)=M_{\delta / 3}\left(\widetilde{B}_{A}(x)\right)$ by (5), it follows that $M_{\delta / 3}\left(\widetilde{B}_{A}(x)\right) \leq c^{\prime}$ where $c^{\prime}=M_{\delta / 3}\left(N_{A}(K)\right)$. Let $D$ be a compact set of $\bar{M}$ and choose $E_{A} \in \mathscr{S}(D, A)$ such that $\# E_{A}=M_{A}(D)$. Then $D \subset \bigcup_{x \in E_{A}} \widetilde{B}_{A}(x)$. Hence we have

$$
\begin{aligned}
M_{\delta}(D) & \leq M_{\delta}\left(\bigcup_{x \in E_{A}} \widetilde{B}_{A}(x)\right) \\
& \leq \# E_{A} \max \left\{M_{\delta / 3}\left(\widetilde{B}_{A}(x)\right): x \in E_{A}\right\} \\
& \leq M_{A}(D) c^{\prime} .
\end{aligned}
$$

We now calculate that

$$
\begin{aligned}
M_{\delta}\left(\widetilde{B}_{\lambda t}\left(y_{0}\right)\right) & \leq M_{\delta}\left(\bar{f}\left(\widetilde{B}_{t}\left(y_{0}\right)\right)\right) \leq c^{\prime} M_{A}\left(\bar{f}\left(\widetilde{B}_{t}\left(y_{0}\right)\right)\right) \\
& \leq c^{\prime} M_{\delta}\left(\widetilde{B}_{t}\left(y_{0}\right)\right)
\end{aligned}
$$

and letting $n=\left[\log _{\lambda} t\right]$

$$
\begin{aligned}
M_{\delta}\left(\widetilde{B}_{c t}\left(y_{0}\right)\right) & \leq c^{\prime} M_{\delta}\left(\widetilde{B}_{c t / \lambda}\left(y_{0}\right)\right) \leq\left(c^{\prime}\right)^{n} M_{\delta}\left(\widetilde{B}_{c t / \lambda^{n}}\left(y_{0}\right)\right) \\
& \leq N\left(c^{\prime}\right)^{n} \quad\left(\text { where } N=M_{\delta}\left(\widetilde{B}_{c \lambda}\left(y_{0}\right)\right)\right) \\
& \leq N\left(c^{\prime}\right)^{\log _{\lambda} t}=N t^{\log _{\lambda} c^{\prime}} .
\end{aligned}
$$

Therefore $\# B(t) \leq N t^{\log _{\lambda} c^{\prime}}$ by $(7)$.

\section{REFERENCES}

1. N. Aoki and K. Hiraide, The linearization of positively expansive maps of tori, The Theory of Dynamical Systems and its Applications to Nonlinear Problems, World Sci. Publ., Singapore, 1984, pp. 27-31.

2. E. Coven and W. Reddy, Positively expansive maps of compact manifolds, Lecture Notes in Math., vol. 819, Springer-Verlag, Berlin and New York, 1980, pp. 96-110.

3. P. Duvall and L. Husch, Analysis on topological manifolds, Fund. Math. 77 (1972), 75-90.

4. J. Franks, Anosov diffeomorphisms, Global Analysis, Proc. Sympos. Pure Math., vol. 14, Amer. Math. Soc., Providence, R.I., 1970, pp. 61-93.

5. M. Gromov, Groups of polynomial growth and expanding maps, Inst. Hautes Études Sci. Publ. Math. 53 (1981), 53-78.

6. R. Kirby and L. Siebenmann, On the triangulation of manifolds and the hauptvermutung, Bull. Amer. Math. Soc. 75 (1969), 742-749.

7. J. Milnor, A note on curvature and fundamental group, J. Differential Geom. 2 (1968), 1-7.

8. M. Shub, Endomorphisms of compact differentiable manifolds, Amer. J. Math. 91 (1969), 175199. 
9. __ Expanding maps, Global Analysis, Proc. Sympos. Pure Math., vol. 14, Amer. Math. Soc., Providence, R.I., 1970, pp. 273-276.

10. J. Wolf, Growth of finitely generated solvable groups and curvature of Riemannian manifolds, J. Differential Geom. 2 (1968), 421-446.

Kagoshima National College of Technology, Shinko 1460-1, Hayato-Cho, AIRA-GUN, KAGOSHIMA 899-51, JAPAN

Current address: Institute of Mathematics, University of Tsukuba, Ibaraki, 305, Japan 